\title{
Present and future management of anti-neutrophil cytoplasmic antibody associated vasculitis: how therapy changed the prognosis
}

\author{
Massimo L'Andolina, ${ }^{1}$ Giovanni Forte, ${ }^{2}$ Norma M. Marigliano, ${ }^{3}$ Salvatore Galasso, ${ }^{4}$ Francesca Mazzei, ${ }^{5}$ \\ Domenico Galasso ${ }^{6}$
}

${ }^{1}$ Ambulatorio di Reumatologia, Divisione di Medicina Interna, PO di Tropea (VV); ${ }^{2}$ Specialista in Malattie dell'Apparato Digerente, Catanzaro; ${ }^{3}$ Specialista in Farmacologia Clinica, Università degli Studi Magna Graecia, Catanzaro; ${ }^{4}$ Specialista in Fisiatria, Mendicino (CS); ${ }^{5}$ Specialista Territoriale in Geriatria, Catanzaro; ${ }^{6}$ Direttore Emerito di Medicina Interna, AO Pugliese Ciaccio, Catanzaro, Italy

\begin{abstract}
Anti-neutrophil cytoplasmic antibody associated vasculitis is part of a multi-systemic idiopathic, small vessel pouci-immune vasculitis. Given the heterogeneous spectrum of the disease, and the need to update therapeutic protocols, the aim of this review was to evaluate clinical-diagnostic approaches. We examined statistical data available in the literature, in particular the 2010 review of St. Hamour et al. Management of Anca-associated Vasculitis, published in Therapeutics and Clinical Risk Management. Acute immunosuppressive therapy and long-term maintenance, with the use of prednisolone, have significantly changed the prognosis of this disease, particularly compared with the 1970s before the introductions of steroids and cyclophosphamide. New drugs such as rituximab, monoclonal antibodies and other modulating immune system molecules are entering clinical use, and experience will confirm whether or not therapeutic guidelines are appropriate. The current diagnostic tools, ranging from laboratory and autoimmune tests, chest X-ray, broncho-alveolar lavage to capillaroscopy, allow prompt diagnosis and early treatment through a first phase of induction-remission, and a second phase of maintenance. There are, however, recurrent and refractory forms of the disease that require long-term immunosuppression and further research into this is merited. These issues have continued to drive the search for safer and more effective modulation of the immune system using targeted immunotherapy. However, the treatment limitations of incomplete efficacy, infection, and cumulative toxicity persist. Modifications to traditional treatment protocols by the use of azathioprine or methotrexate rather than cyclophosphamide, and the introduction of newer agents, such as rituximab, have meant that outcomes have been maintained while toxicity has been reduced.
\end{abstract}

\section{Introduction}

Systemic vasculitis is a heterogenous group of diseases. From a histological perspective, they are characterized by the presence of phlogistic and necrotic phenomena that affect the vessel wall with subsequent ischemia of the interested tissues. ${ }^{1,2}$ Identification of the various forms of vasculitis takes into account the

Correspondence: Domenico Galasso, Viale Brutium 46/A, 88100 Catanzaro, Italy

Tel. +39.347.2561683 - +39.0961.751877.

E-mail: domenico.galasso@libero.it

Key words: vasculitis, Wegener's disease, Churg-Strauss syndrome.

Conflict of interests: the authors declare no potential conflict of interests.

This work is licensed under a Creative Commons Attribution NonCommercial 3.0 License (CC BY-NC 3.0).

(C) Copyright M. L'Andolina et al., 2013

Licensee PAGEPress, Italy

Italian Journal of Medicine 2013; 7:14-25

doi:10.4081/itjm.2013.14 classification proposed at the Chapel Hill Consensus Conference (CHCC). ${ }^{3}$ This classification divides vasculitis into: vasculitis of small-medium-large caliber vessels. The involvement of small and medium caliber vessels is typical of anti-neutrophil cytoplasmic antibody (ANCA) associated vasculitis (AAV). These belong to the idiopathic multi-system, small vessel, pouci-immune vasculitis. These are rare pathologies. With respect to the general population, patients with vasculitis have a worse prognosis with reduced survival (Figure 1). The annual incidence in Europe is estimated at 9.7 cases per 1,000,000 inhabitants for Wegener's granulomatosis, 2.7 cases for microscopic polyangiitis and 2.7 for Churg-Strauss.

\section{Pathogenesis}

This pathology is characterized by the presence of autoantibodies called ANCA. These subsequently release enzymes capable of causing cell damage and, therefore, inflammation. The autoimmune response involves various types of cells: cells with antigens, $\mathrm{T}$ lymphocytes, neutrophils and B lymphocytes. These cells are responsible for ANCA synthesis, the intimate pathogenetic sequence, although this mechanism is 
still not clear. There is an initial pathogenetic intereaction between neutrophils and endothelial cells. Infection is a trigger factor as is exposure to other environmental stimuli that result in pro-inflammatory cytokine secretion that can cause the migration of some proteins of the neutrophil granules to the cell surface. Some of these can function as antigen for the ANCA interacting with the cell surface antigens, PR3 or MPO, and the FC receptor. In this phase, the neutrophils adher to the endothelial depositing molecules that are reactive to oxygen, proteolytic enzymes and factors that activate a complementary pathway causing damage to the endothelial and other vessel walls.

ANCA neutrophils, activated in turn, deposit other pro-inflammatory cytokines that attract other neutrophils and cells that augment the process. The monocytes, activated in the same way, can also contribute to the inflammatory process as macrophages. The damage to the vessel wall is a result of various factors, from direct toxic effects, to the expression of foreign antigens on the endothelial cells with consequent stimulation of the cell-mediated or humoral immunitary response. The result is that the activated ANCA neutrophils and other inflammatory cells, such as lymphocytes and monocytes, infiltrate and destroy the blood vessel wall. The inflammatory and necrotizing process can also affect the perivascular tissue.

A certain genetic disposition for the pathology is recognized, expressed by the significant association with particular histocompatible antigens (HLA DR1, DR2, DQW7). The damage to the vessel wall, according to the organ affected, can cause hemorrhage (hematuria, hemoptysis), thrombotic phenomena, narrowing of the vessels reducing blood flow and causing organ damage. The organs most affected are the kidney and lung but other lesions can also be observed on the skin, eyes and other vessels. The ANCA (cANCA and p-ANCA) can be identified by indirect immunofluorescence. The various clinical entities are: Wegener's granulomatosis (WG), Churg-Strauss syndrome (CSS), microscopic polyangiitis (MPA), limited renal vasculitis. ${ }^{4-6}$ The European Vasculitis Study Group was set up to investigate the pathogenesis of these diseases and to define optimal better targeted and less toxic therapeutic protocols.

\section{Wegener's granulomatosis}

Prevalence of $\mathrm{WG}$ in the adult population is $3: 100,000$. The male to female ratio is $2: 1$. Median age is 45 years and, although it can present at any age, it is most frequently seen in patients $35-55$ years old. ${ }^{7,8}$ Some authors have proposed modifying the term Wegener's granulomatosis to Wegener's polyangiitis. ${ }^{9}$ The seriousness of the disease is related to kidney involvement, seen in $75 \%$ of patients. Five-year survival after treatment is $95 \%$. In contrast, if the disease is not treated, 5 -year survival is $90 \% .^{10,11}$

\section{Signs and symptoms}

Non-specific symptoms are fever, anorexia, general malaise, arthralgia, myalgia, weight loss. Involvement of the respiratory tract can include chronic sinusitis that does not respond to treatment and chronic otitis with the loss of hearing, and ulceration of the nasal and oral mucosa.

Nasal obstruction is due to thickening of the mucosa with significant bone lesions or ulcerating lesions that destroy soft tissue, extremely thick scabs with perforation of the nasal septum, the collapse of the root of the nose, deformity of the nose to a saddle shape, purulent nose bleeding, susceptibility to infection. Sixty-three percent of patients present chronic nasal colonization of Staphylococcus aureus compared with $20 \%$ of healthy subjects, and this is significantly associated with recurrence.

In the ear, nose and throat, WG can be aggressive with extensive necrosis and collapse of the nasal bridge, epistasis, conductive deafness, and subglottic stenosis.

In orbital disease in WG, conjunctivitis and scleritis can also be observed as can retro-bulbar proptosis of granuloma. Prognosis is reserved if necrotizing scleritis is also presented. Hyperplasia and gingival petechiae can also be observed.

On the skin, cutaneous ulcerations and vesicles are found in $13 \%$ of patients. Palpable purpura, papules, subcutaneous nodules, petechiae, pustules, hemorrhagic bullae, digital necrosis, sub-ungual hemorrhage, genital ulcers and xanthelasma can also be observed (Figures 2 and 3). ${ }^{12,13}$

The lungs are involved in $85 \%$ of cases with hematosis, cough, dyspnea and chest pain. There is nodular opacity and this is usually multiple of variable

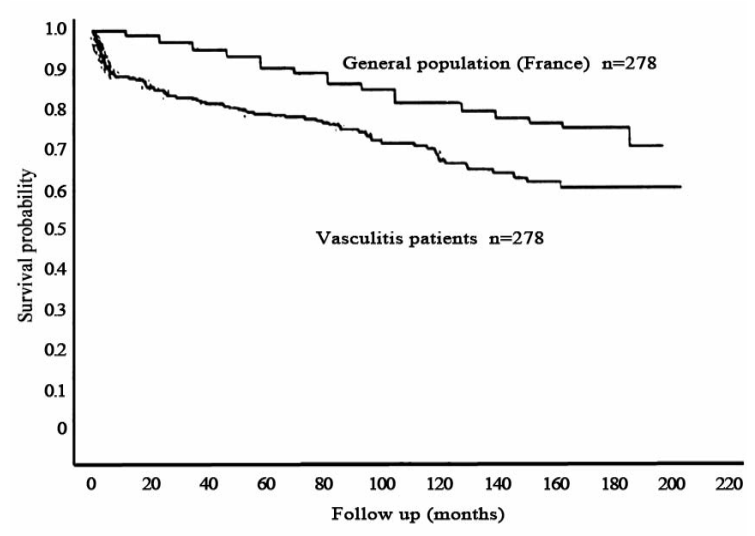

Figure 1. Survival of healthy subjects and associated vasculitis patients. Adapted from Brown, 2006. ${ }^{15}$ 
diameter from just a few millimeters to 9 centimeters. This is often found in the sub-pleuric site and in 50\% of cases results in cavitation. Asymptomatic lung involvement is common (30\%). In $5 \%$, clinical onset is fulminant. Infiltrates resulting from the increase in parenchymal density can be mono, bilateral, diffuse or patchy. There is concomitant thickening of the interlobular septa and pleural effusion in $20 \%$ of patients (Figures 4 and 5).

Seventy-five percent of patients have renal involvement and this is sustained by the presence of focal and segmentary necrotizing glomerulonephritis. Hematuria and oliguria can appear ${ }^{14,15}$ or frank proteinuria with kidney failure due to the insurgence of a fulminant diffuse necrotizing glomerulonephritis (Table 1). ${ }^{15,16}$

The clinical triad that characterizes WG is due to involvement of the upper respiratory tracts, the lower respiratory tracts and to glomerulonephritis $(40 \%$ at initial presentation). ${ }^{17,18}$ Wegener's granulomatosis is associated to c-ANCA positivity (PR3) in approximately $80 \%$ of cases. Figure 6 shows by indirect immunofluorescence on neutrophils fixed in ethanol (ANCA) the pattern of cytoplasmic coloring could be c-Anca (PR3), with a worse prognosis, or perinuclear, p-Anca (MPO) (Figure 7).

In such cases, other organs are also often involved. There is frequently a quicker decline in renal function and a tendency towards recurrence; all markers of a possible worsening of the clinical condition. Other clinical manifestations include involvement of the pe-

Table 1. Clinical characteristics in Wegener's granulomatosis.

\begin{tabular}{lc}
\hline Clinical symptoms and signs & Prevalence (\%) \\
\hline $\begin{array}{l}\text { Pulmonary (cough, dyspnea, chest pain, } \\
\text { hemoptysis) }\end{array}$ & $70-95$ \\
\hline $\begin{array}{l}\text { Upper airway (epistaxis, rhinorrhea, sinusitis, } \\
\text { otitis, hearing problems, ear pain, destructive } \\
\text { lesions/bone deformities, ulcerations) }\end{array}$ & $70-95$ \\
\hline $\begin{array}{l}\text { Tracheobronchial (subglottic stenosis, } \\
\text { bronchial stenosis, endobronchial lesions) }\end{array}$ & $10-55$ \\
\hline \begin{tabular}{l} 
Kidney damage, glomerulonephritis \\
\hline $\begin{array}{l}\text { Cutaneous (purpura, nodules, vesicles, ulcers) } \\
\text { Musculoskeletal (myalgias, arthralgias, arthritis) }\end{array}$
\end{tabular} & $45-60$ \\
\hline $\begin{array}{l}\text { Ocular (conjunctivitis, uveitis, episcleritis, } \\
\text { scleritis, proptosis) }\end{array}$ & $25-55$ \\
\hline $\begin{array}{l}\text { Non-specific, constitutional (malaise, fever, } \\
\text { weight loss, arthralgias) }\end{array}$ & $15-45$ \\
$\begin{array}{l}\text { Peripheral and central nervous system, } \\
\text { headache }\end{array}$ & $10-30$ \\
\hline $\begin{array}{l}\text { Cardiovascular (coronary vasculitis, } \\
\text { pericarditis) }\end{array}$ & $5-15$ \\
\hline
\end{tabular}

Adapted from Brown, 2006. ${ }^{15}$ ripheral nervous system, symmetric polyneuropathy and multiple mononeuritis. It is also possible to observe involvement of the gastrointestinal tract in the form of enterorrhagia, increase in hepatic enzymes, diarrhea, nausea, vomiting, and abdominal pain.

Finally, manifestations in the joints are possible. These range from simple arthralgia to true and proper

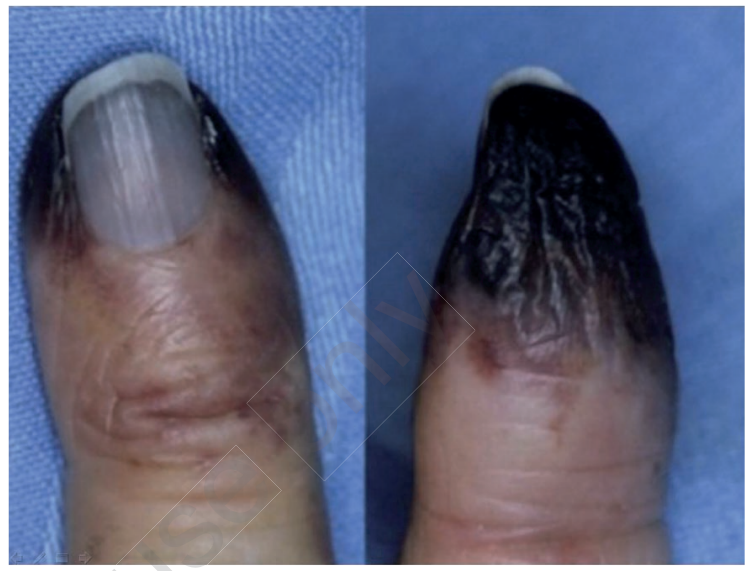

Figure 2. Necrotizing vasculitis (Wegener's granulomatosis) involving small and medium vessels.

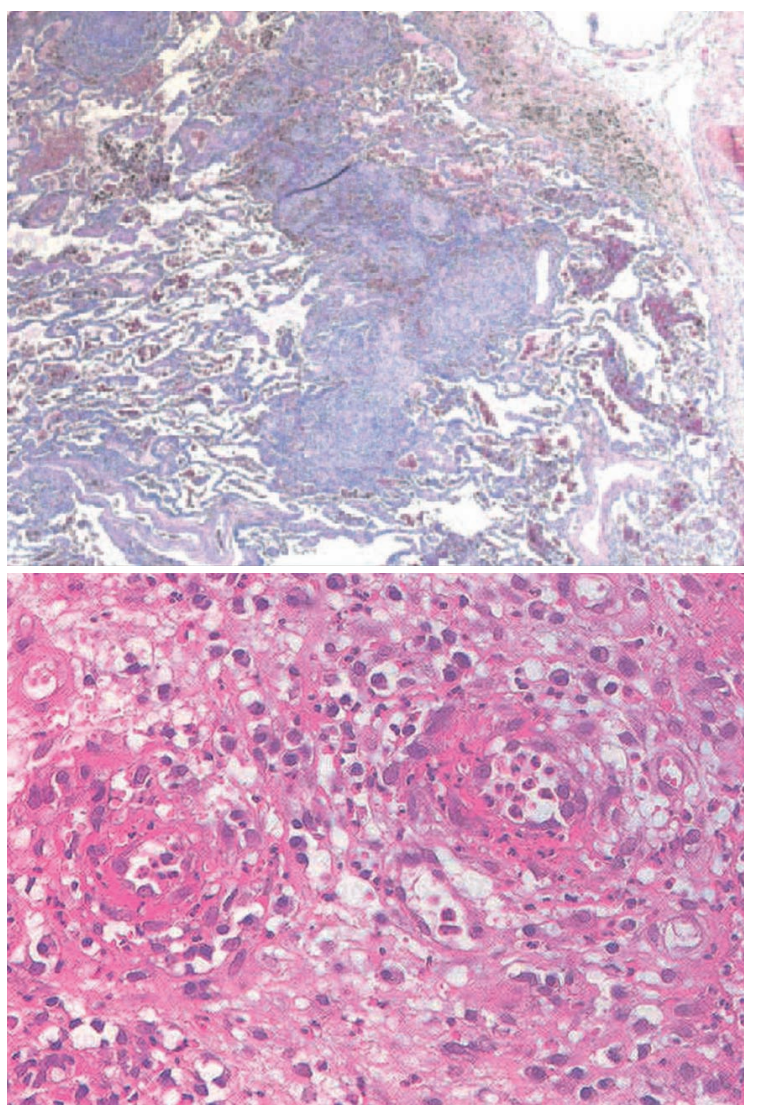

Figure 3. Necrotizing granulomatous vasculitis. 
oligo-polyarthritic phenomena. Heart problems such as pericarditis, coronary vasculitis or, rarely, cardiomyopathy, are found in $8 \%$ of patients.

ANCA negativity indicates a localized disease (Tables 2 and 3). ${ }^{19,20}$ Positivity (p-Anca, MPO) is found in $20-25 \%$ of active cases. VES is raised. Azotemia and creatinine are increased with changes in urinary sedimentation in the case of renal involvement. If rheumatoid screening is positive, the complement is not consumed and antinuclear antibodies are absent. IgA are increased and sometimes total IgE and immune complexes. ${ }^{21}$ Instrumental tests are useful. Non-invasive tests include chest X-ray that can show lung infiltrates and granulomas. Also useful are X-ray of the paranasal sinuses, with evident sinusitis, and invasive tests such as skin (aspecific), kidney and lung biopsy. This latter is a more useful diagnostic tool than skin biopsy. There are numerous differential diagnoses such as lethal franuloma with lymphomatoid granulomatosis, Churg-Strauss syndrome, microscopic polyangiitis, carcoidosis, pulmonary granulomatose infective diseases

Table 2. Role of c-ANCA (PR3-ANCA) and p-ANCA anti-MPO.

\begin{tabular}{lcc}
\hline ANCA-associated vasculitis & \multicolumn{2}{c}{ Sensitivity (\%) } \\
\hline & PR3-ANCA & MPO-ANCA \\
\hline Wegener's granulomatosis & $70-80$ & 10 \\
\hline Microscopic polyangiitis & 30 & 60 \\
\hline $\begin{array}{l}\text { Evolution of idiopathic } \\
\text { glomerulonephritis }\end{array}$ & 30 & 64 \\
\hline Churg-Strauss syndrome & $<5$ & 40 \\
\hline
\end{tabular}

ANCA, anti-neutrophil cytoplasmic antibody; PR3, anti-proteinase 3; MPO, myeloperoxidase.

Table 3. Positive ANCA-associated diseases and target antigen.

\begin{tabular}{lccc}
\hline $\begin{array}{l}\text { Systemic } \\
\text { vasculitis }\end{array}$ & $\begin{array}{c}\text { ANCA } \\
\text { positive }\end{array}$ & \% & $\begin{array}{c}\text { Target } \\
\text { antigen (\%) }\end{array}$ \\
\hline $\begin{array}{l}\text { Wegener's } \\
\text { granulomatosis } \\
\text { (generalized forms) }\end{array}$ & c-ANCA & $80-90$ & PR3 (70-80) \\
\hline Microscopic polyangiitis & p-ANCA & $80-85$ & PR3 (20-30) \\
\hline $\begin{array}{l}\text { Churg-Strauss syndrome } \\
\text { c-ANCA }\end{array}$ & 50 & PR3 (30-40) \\
\hline $\begin{array}{l}\text { Classic polyarteritis } \\
\text { nodosa }\end{array}$ & p-ANCA & 10 & MPO (90) \\
\hline
\end{tabular}

ANCA, anti-neutrophil cytoplasmic antibody; PR3, anti-proteinase 3; MPO, myeloperoxidase. Adapted from Occhipinti and Bartolomei, 2008. ${ }^{20}$

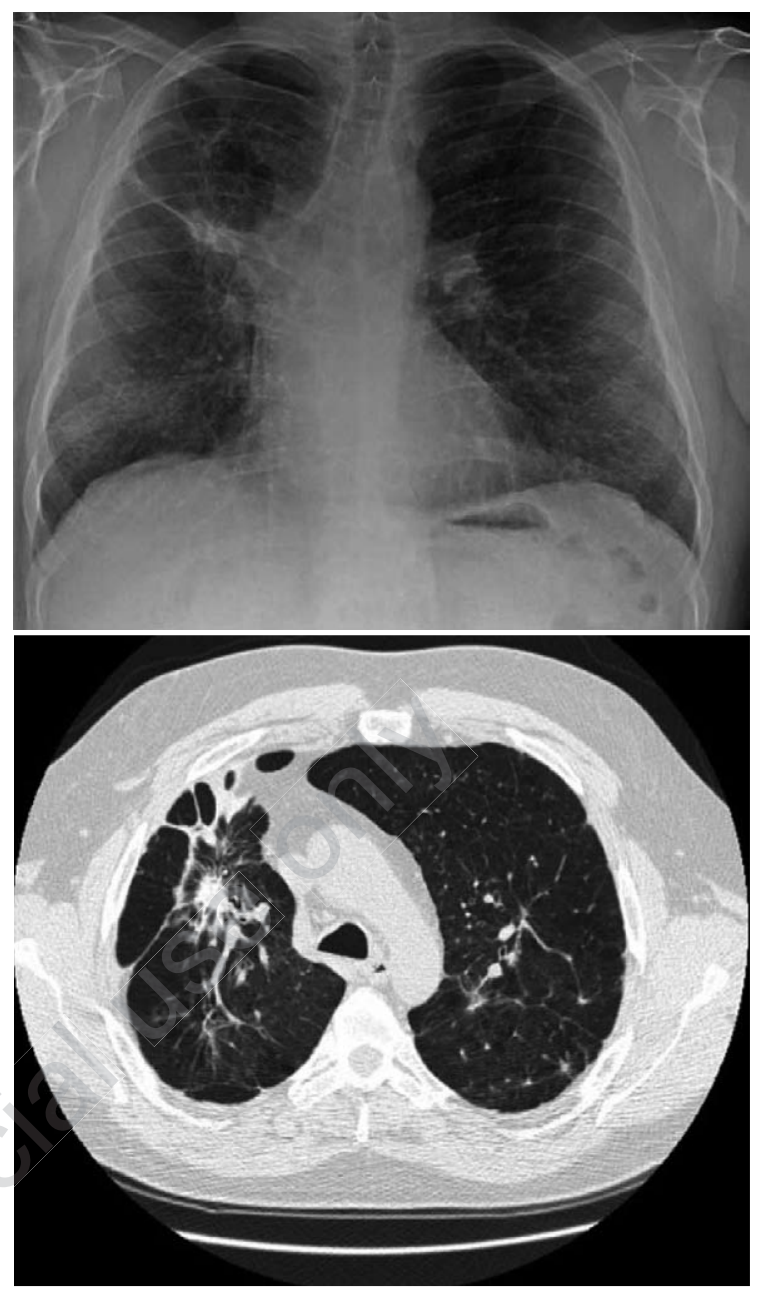

Figure 4. Infiltrates of increased bilateral parenchymal density, mainly on DX; diffused and patchy. Interlobular septum thickening.

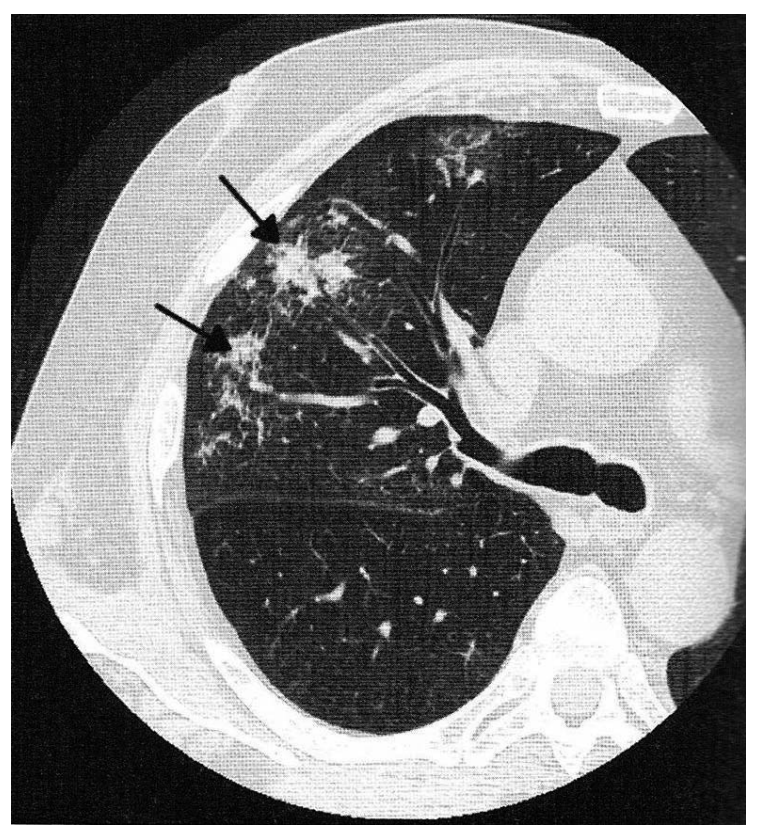

Figure 5. Patchy infiltrates. 
(tuberculosis, fungal infections), Goodpasture syndrome, lung cancer, Henoch-Schönlein syndrome and pyoderma gangrenosum.

\section{Diagnostic criteria of the American College of Rheumatology}

The American College of Rheumatology (ACR) defined the following diagnostic criteria: i) nasal or oral inflammation, appearance of painful, non-painful or purulent mouth ulcers, epistasis, hemoptysis, during active disease; ii) abnormal radiographic findings of the chest (with reference to the lung), presence of nodules, fixed or cavity infiltrates; iii) urinary microhematuria over 5 red blood cells per field, or erythrocyte cylinders in the urinary sediment; vi) biopsy showing granulomatose inflammation inside the arterial wall or in the perivascular or extravascular area.

Diagnosis is based on satisfying 2 of these 4 criteria. If 2 or more criteria are satisfied, sensibility is confirmed at $88.2 \%$ and specificity at $92.0 \%$.

\section{Churg-Strauss syndrome}

Granulomatous lung disease may be difficult to distinguish from mycobacterial infection. ChurgStrauss syndrome is a systemic disorder characterized by asthma, transitory pulmonary infiltrates, hypereosinophilia and systemic vasculitis. ${ }^{22,23}$ The male to female ratio is 1.3:1. The median age at onset is around 40 years.

The disease develops in three distinct phases that can be sequential: i) allergic with cough, asthma, rhinitis, sinusitis, cephalea, nasal polyps; ii) eosinophilia with weight loss, physical weakness, chest pain, dyspnea, abdominal pain, diarrhea, fever, sweating, cough, hemoptysis, ascites, intestinal bleeding, presentation of abdominal mass, intestinal occlusion, arthro-myalgie; iii) vasculitic phase with fever, weight loss, lymphodenopathy, presentation of gallop rhythm, pericardial friction, turgid jugular, peripheral edema, peripheral neuropathy (with glove and sock distribution). The sudden onset of wrist or foot (equine foot) extension deficit is often caused by vasculitis. Furthermore, we can also find loss of acuity, brain involvement (hemorrhage/heart attack), hematuria and proteinuria, multiform erythema, petecchie, extensive ecchymosis, cutaneous nodules, facial edema, livedo reticularis, itching and vesicles. Such clinical findings would constitute a negative prognosis. ${ }^{24}$

Allergic rhinitis or bronchial asthma are frequent and these can precede the vasculitis also by many years. Allergic rhinitis or bronchial asthma worsen on the onset of vasculitis, characterized by systemic manifestations such as fever, weight loss, asthenia. If respiratory symptoms are also present they are modest with a scarsely productive cough and dyspnea. Diffuse or temporary patchy infiltrates are a typical expression of diffuse interstitial pulmonary disease. Sometimes, bilateral nodules, some centimeters in dimension, are presented that, in contrast to that seen in Wegener's granulomatosis are not deep. Churg-Strauss syndrome is associated to MPO-ANCA positivity, according to

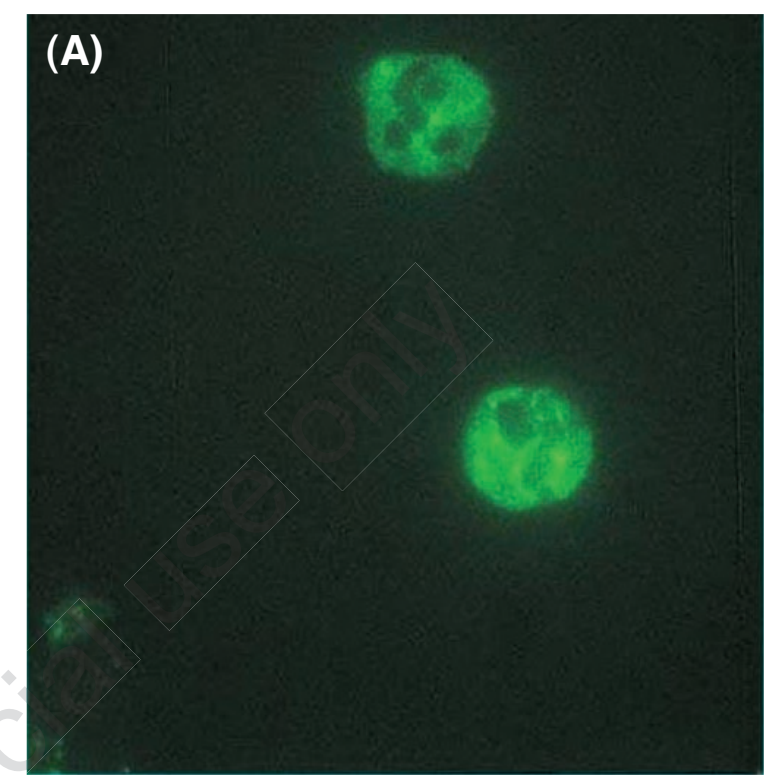

\section{(B)}

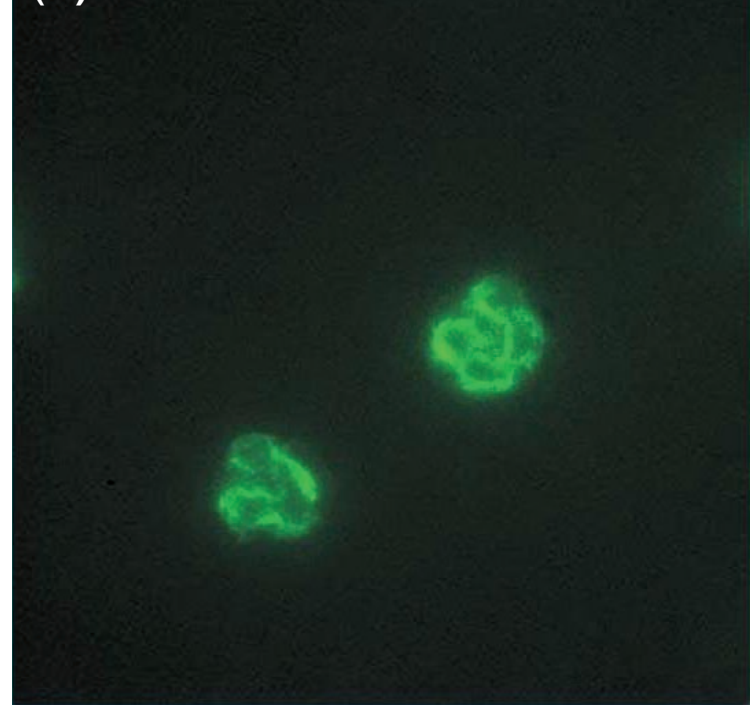

Figure 6. Indirect immunofluorescence on neutrophils fixed in ethanol (anti-neutrophil cytoplasmic antibody, ANCA). Pattern of cytoplasmic (cANCA) or perinuclear (pANCA) coloring. (A) Anti-proteinase 3. Cytoplasmic coloring is due to autoantibodies directed against the anti-proteinase $3 \mathrm{c}$ ANCA (PR3-ANCA). C-ANCA 80\% Wegener's granulomatosis. (B) Anti-myeloperoxidase. Perinuclear coloring is due to autoantibodies directed against the myeloperoxidase p-ANCA (MPO-ANCA). P-ANCA 60\% microscopic polyangiitis-Churg-Strauss syndrome. 
Kulleberg by $40 \%$, and associated to c-ANCA, PR3 positive in less than $5 \%$; according to other authors, Occhipinti by $60-70 \%$ for MPO and $30-40 \%$ per PR, or to ANCA negativity but with infiltration of eosinophils in the tissue. ${ }^{25}$ The possibility of variable and multiple clinical profiles, and numerous similar signs and symptoms, makes diagnosis difficult given the similarities with common pathologies such as infections, neoplasia, TEV, connective tissue disease, pharmacological toxicity, sarcoidosis, pulmonary interstitial disease. Suspicion of characteristic clinical conditions requires complete and aggressive diagnostic evaluation. ${ }^{26}$ Possible survival with respect to the general population is reduced in patients with vasculitis. ${ }^{27}$ Principal causes of morbidity and mortality are myocarditis and myocardial infarction, resulting from coronary arthritis. One-year survival of treated patients is $90 \%$; 5 -year survival for these patients is $62 \%$.

It has been established that a diagnosis of ACR requires at least 4 of the following criteria: asthma, eosinophilia over $10 \%$, mononeuropathia or polyneuropathia, temporary pulmonary infiltrates, sinusitis and extravascular eosinophils (on arteriole, arterial, and/or venous biopsy). Renal vasculitis can be limited and can cause asymptomatic glomerulonephritis pauci or, on the other hand, can present in a diffuse active form leading to a reserved prognosis. Serious complications include onset of rapidly progressive glomerulonephritis and pulmonary hemorrhage. Limited disease refers to patients with AAV and one or more involved organ systems, but no renal disease or immediately life-threatening complications (Table 1). The intensity (and, therefore, tolerated potential toxicity) of treatment depends on disease severity.

In clinical practice it is useful to apply a score capable of defining the seriousness of the disease, such as the Birmingham Activity Vasculitis Score (this evalutes both disease activity and its severity) ${ }^{19,28}$ and the Vasculitis Damage Index that evaluates the extent of vascular damage.

\section{Other clinical characteristics}

\section{Diffuse alveolar hemorrhage}

Diffuse alveolar hemorrhage (DAH) has an incidence of $7-45 \%$ in WG, $10-30 \%$ in MPA. It is rare in CSS. The clinical profile is characterized by dyspnea, fever, cough, hemoptysis, cyanosis, acute respiratory stress syndrome. This is a serious, potentially catastrophic complication. The immunocomplexes deposit in the alveolar septa and in the blood vessels, activating the complement and breaking the integrity of the basal capillary alveolar with hemorrhage. There are also signs of capillaritis. Survival is often severely compromised (50-70\%). Rapidly progressing glomerulonephritis rep- resents approximately $5 \%$ of all causes of kidney failure. Important clinical signs are hematuria, proteinuria $(>500 \mathrm{mg} / \mathrm{d})$, hyperazotemia, raised creatinine levels, edema and arterial hypertension.

\section{Microscopic polyangiitis}

This is a necrotizing non-granulomatous systemic vasculitis. It was first thought to be a variation of polyarteritis nodosa (PAN). In 1994, on the basis of clinical and histopathological criteria, the CHCC definitively distinguished between the two diseases. Median age at onset is 50 years. The following are nearly always presented: segmentary focal necrotizing glomerulonephritis, and pulmonary capillaritis. The overt phase of the disease can be preceded by months or even years by systemic symptoms (arthralgia, general malaise, fever, weight loss) with increased phlogosis indexes and anemia. Sometimes the initial involvement is only renal. Pulmonary manifestations are characterized by hemoptysis, pulmonary hemorrhage $(12-29 \%)$ caused by alveolar capillaritis, or, more rarely by rupture of the pulmonary artery. Recurrence is common (11-46\%) subsequent to some years of temporary focal infiltrates, exudate or fibrinous pleurisy, pulmonary fibrosis. Prognosis is extremely poor.

A diagnosis of MPA is confirmed if anti-myeloperoxidase ANCA (MPO-ANCA) is found in the serum, present in $51-75 \%$ cases. The presence of these and the absence of microaneurisms and/or stenosis of the

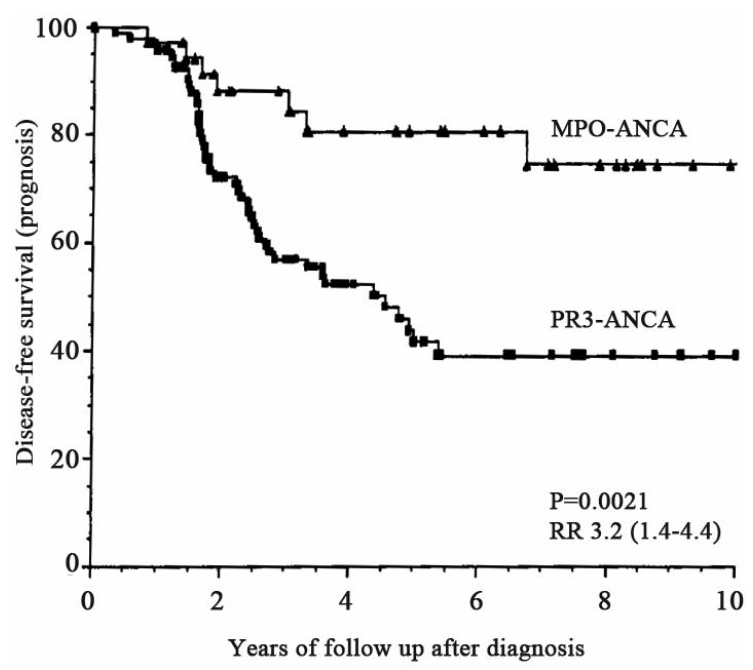

Figure 7. Prognosis of patients with myeloperoxidase pANCA and anti-proteinase 3 c-ANCA. Adapted from Rutgers et al., 2003. ${ }^{23}$ 
renal vessels is useful in differential diagnosis with PAN. C-ANCA positivity, anti PR3 is found in 10$40 \%$ of cases and is associated with a worse prognosis. In some cases, ANCA are absent.

\section{Diagnostic methods}

High-resolution pulmonary computerized tomography is a very important diagnostic tool. It shows bilateral ground glass opacity mainly distributed in patches and in the sub-pleural site, centrolobular pulmonary nodules $(<5 \mathrm{~cm})$ often in ground glass opacity, thickening or dilation of the bronchial wall and of the interlobular septa, normal findings, signs of vasculitis, exudate pleuritis, diffuse alveolar hemorrhage. Chest imaging in ANCA-associated vasculitis can shown many non-diagnostic findings (Figure 4). Nodules and/or excavations are more specific in patients with Wegener's granulomatosis (Figure 5). Nodular pathology is found in $55-70 \%$ and cavitary symptoms in 35$50 \%$, and these should be included in differential diagnosis with infections and tumors. ${ }^{29}$

Broncho-alveolar lavage (BAL) shows marked eosinophilia (4-66\%). The BAL eosinophilia and $\operatorname{IgE}$ levels correlate with the disease activity more than peripheral eosinophilia. Alveolar eosinophilia can be observed also in patients with normal chest X-ray and respiratory function. Disease activity and severity may be assessed using the well-characterized Birmingham Vasculitis Activity Score ${ }^{11}$ while disease-induced damage is measured by the Vasculitis Damage Index. ${ }^{12}$

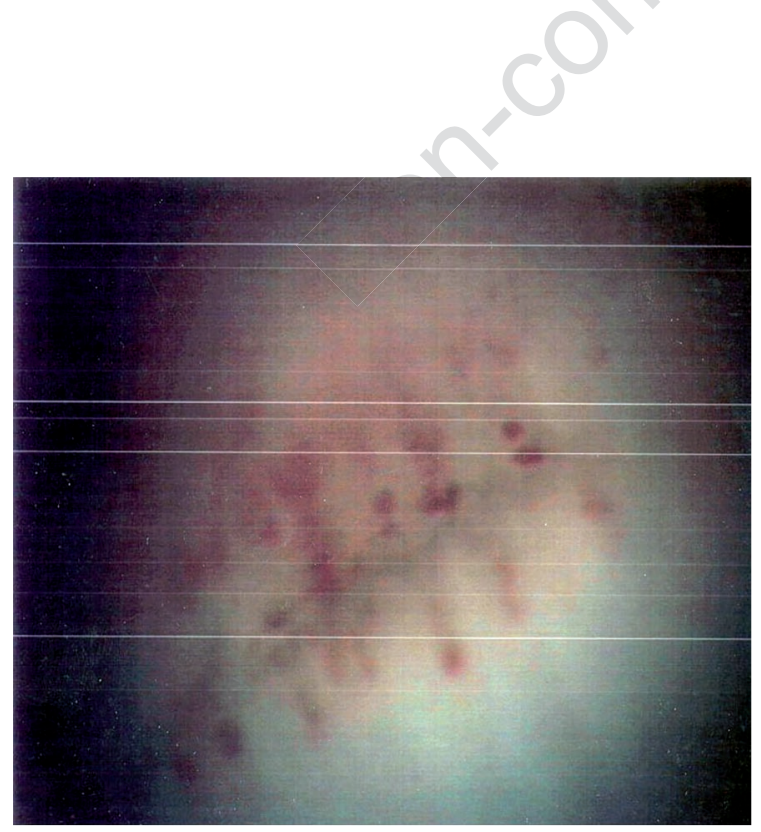

Figure 8. Capillaroscopy imaging: marked tortuosity of capillary loops, slight or medium ectasia, microhemorrhage.
These scores have been mostly used as research and clinical trial tools enabling comparison of patients, but may also be used to facilitate patient management.

The capillaroscope has no diagnostic role and can show both forms: marked tortuosity of the capillary loops, slight or medium extasia, microhemorrhage in $25-30 \%$ of cases, cytoarchitectonic study shows conserved comb capillary. Neoangiogenesis is rare and almost never megacapillary. In WG, neoangiogenesis can reach $50 \%$ (Figure 8 ).

\section{Treatment}

A prompt diagnosis is essential and immunosuppression should be started immediately according to the seriousness of the clinical profile. Treatment goes from induction-remission therapy to maintenance, with careful monitoring of drugs and disease course. Before the introduction of corticosteroids and immunosuppressors, patients with vasculitis, and particularly those with WG, had an average survival of only five months. The introduction of steroids has only slightly changed the prognosis ( 8 months) although the introduction of cyclophosphamide in the 1970s was important.

The EUVAS has presented therapeutic guidelines, distinguishing limited forms of disease, with serum creatinine $<1.4 \mathrm{mg} / \mathrm{dL}$, and treatment with corticosteroids or methotrexate or azatioprine. The generalized early form with serum creatinine $<1.4 \mathrm{mg} / \mathrm{dL}$ can take advantage of combined treatment with corticosteroids and cyclophosphamide or corticosteroids and methotrexate. The generalized active form with serum creatinine $>5-7 \mathrm{mg} / \mathrm{dL}$ requires corticosteroids, cyclophosphamide and, in the severe form, plasmapheresis is added. Five-year mortality is 50\% with only steroids and falls to $12 \%$ with the addition of cyclophosphamide (Tables 4 and 5). ${ }^{2}$ Cortisones are administered at high doses (prednisone 1 $\mathrm{mg} / \mathrm{kg} / \mathrm{die}$ ) and cyclophosphamide $2 \mathrm{mg} / \mathrm{kg} / \mathrm{die}$. Endovenous bolus cyclophosphamide $0.6 \mathrm{~g} / \mathrm{m}^{2}$ is also used once a month.

\section{Treatment of anti-neutrophil cytoplasmic antibody associated vasculitis}

This can be divided into two phases. The first is induction-remission and the second is maintenance. There is a wide variability in WG evolution that requires frequent modifications to the drugs used according to the disease phase and activity. Other important factors are recognition and treatment of possible remission, therapeutic approach in the resistant forms, in patients undergoing dyalisis or following transplant, and in pregnant women. ${ }^{30}$

The current induction treatment most frequently 
used is $2 \mathrm{mg} / \mathrm{kg} /$ die oral cyclosphamide (Cyp), and corticosteroids, usually prednisone, at an oral dosage of $1 \mathrm{mg} / \mathrm{kg} / \mathrm{die}$ (or equivalent). The corticosteroids are administered to rapidly reduce inflammation until Cyp starts to take effect (after approx. 1-2 weeks). Prednisone (PD) is taken for at least four weeks. Once patient condition improves, this can be gradually reduced over 1-2 months by 5-10 mg a week. A maintance therapy can then be established of $10-15 \mathrm{mg} / \mathrm{die}$ that can be further reduced, first to alternate days and then suspension of treatment with oral Cyp as only maintenance. The length of the regime with alternate days of PD administration can vary according to individual patient response. ${ }^{31}$

Induction of remission using oral Cyp is achieved within six months with a therapeutic response in around $90 \%$ of cases, and complete remission in $75 \%$ of these. According to some authors, Cyp should be continued for at least a year after clinical remission is achieved. Dosage should then be gradually reduced by $25 \mathrm{mg}$ every 2-3 months until therapy is suspended. There is a high percentage of relapse and side effects from the drugs used. Higher doses of Cyp (3-5 $\mathrm{mg} / \mathrm{kg} / \mathrm{die}$ ) can be administered for some days in the more severe and rapidly progressive forms of disease with important renal and pulmonary involvement. In these cases, it is also possible to start treatment with endovenous bolus methylprenisolone (from 250 to $1000 \mathrm{mg}$ ) for three consecutive days. Combined oral therapy with Cyp and PD is, however, associated with a high level of toxicity. There is a high risk of myelotoxicity and incidence of amenorrhea in around 50\% of cases. Other side effects are hemorrhagic cystitis $(15 \%)$, cancer of the bladder $(5 \%)$, myelodysplasia (2\%) and lymphoma (1\%), opportunitistic infection, Pneumocystis carinii pneumonia, cataracts, diabetes mellitus, osteopenia, fracture and aseptic osteonecrosis. ${ }^{32}$ As a possible alternative strategy, to reduce the overall cumulative dose of Cyp, monthly endovenous bolus of Cyp at an initial dose of $0.5 \mathrm{~g} / \mathrm{m}^{2}$ of body surface can be indicated, increased up to $1 \mathrm{~g} / \mathrm{m}^{2}$. It has been proposed that in cases refractory to Cyp, initial forms of this disease could be treated with a safer therapy such as methotrexate (MTX). This is a folic acid analog that inactivates the dihydrofolate enzyme reductase and, therefore, production of thymidylate, essential for DNA replication. Treatment should be started with weekly oral or intramuscular doses of 0.3 $\mathrm{mg} / \mathrm{kg}$ and can be gradually increased by $2.5 \mathrm{mg}$ a week up to the maximum dose tolerated (however, not over $25 \mathrm{mg}$ /week).

Folic acid (1-2 mg/die) or folinic acid (2.5-5 $\mathrm{mg} /$ week, $24 \mathrm{~h}$ after administration of MTX) can be given to prevent some of the drug's side effects. In approximately $70 \%$ of cases, remission is achieved at six months and in $90 \%$ at one year. Thirty-five to $70 \%$ of cases relapse.

Another possible therapeutic option to induce re-

Table 4. European Vasculitis Study Group (EUVAS) grading of disease severity and first-line treatment options for induction therapy.

\begin{tabular}{lcccc}
\hline Classification & Symptoms & Renal function & $\begin{array}{c}\text { Compromized } \\
\text { functionality }\end{array}$ & Therapeutic options \\
\hline Limited & No & Serum creatinine $1.4 \mathrm{mg} / \mathrm{dL}$ & No & Corticosteroids or MTX or AZA \\
\hline Early, generalized & Yes & Serum creatinine $1.4 \mathrm{mg} / \mathrm{dL}$ & No & Cyp+corticosteroids or MTX+corticosteroids \\
\hline Active, generalized & Yes & Serum creatinine $5.7 \mathrm{mg} / \mathrm{dL}$ & Yes & Cyp+corticosteroids \\
\hline Severe & Yes & Serum creatinine $5.7 \mathrm{mg} / \mathrm{dL}$ & Yes & Cyp+corticosteroids+plasma exchange \\
\hline
\end{tabular}

MTX, methotrexate; AZA, azatioprine; Cyp, cyclosphamide.

Table 5. Therapeutic strategies.

\begin{tabular}{lccc}
\hline Therapy & \multicolumn{1}{c}{ Drug } & Dose & Duration \\
\hline Induction therapy & Cyp & $2 \mathrm{mg} / \mathrm{kg}$ according to renal function and if age $>60$ years & $3-6$ months \\
\cline { 2 - 4 } & Prednisolone & $1 \mathrm{mg} / \mathrm{kg}$ \\
$10-15 \mathrm{mg} / \mathrm{kg}$ & $\begin{array}{c}4 \text { weeks, staggered dosage } \\
\text { After 4 weeks and up till } 6^{\text {th }} \text { month }\end{array}$ \\
\hline Adjuvant therapy & $\begin{array}{c}\text { Plasmapheresis } \\
\text { methylprednisolone }\end{array}$ & $1 \mathrm{~g}$ & $2-3$ weeks \\
\hline Maintenance therapy & AZA & $2 \mathrm{mg} / \mathrm{kg}$ & 24 months \\
\cline { 2 - 4 } & Prednisolone & $7.5 \mathrm{mg}$ & $7-24$ months \\
\hline
\end{tabular}

AZA, azatioprine; Cyp, cyclosphamide. Adapted from Kallenberg, $2007 .^{2}$ 
mission in WG that is recommended for all those forms of vasculitis with severe renal involvement is plasmapheresis. This can be used in addition to other therapies in an attempt to quickly normalize the immune system and, therefore, prevent rapid evolution and unfavorable prognosis. Data available in the literature provide contrasting data. The reported improvement following apheresis treatment does not, in fact, in the short term correspond to any substantial difference in survival of these patients, nor in the general disease evolution. In any case, there seem to be three distinct subsets of WG patient who could benefit from apheresis treatment: i) patients with anti-membrane basal glomerular anti-bodies (anti-GBM Ab); ii) patients with dialysis-dependent kidney failure on onset of disease; and iii) those with severe pulmonary hemorrhage.

\section{Maintenance therapy}

In the past, cytotoxic therapy with Cyp and PD was continued for at least one year after achieving complete clinical remission. Currently, other immunosuppressors provide effective protection against relapse and are less toxic. Switching to one of these therapies is, therefore, recommended as soon as remission is stabilized.

Sometimes low doses of corticosteroids, also on alternate days, are used as maintenance therapy together with cytotoxic drugs. On average, steroid therapy continues for a period of from around a year to 7-8 months in order to avoid any possible side effects.

In the long term, Cyp can be replaced first with MYX and, in patients with kidney failure, with azatioprine (AZA). Low-doses of $0.3 \mathrm{mg} / \mathrm{kg}$ once a week have been seen to be effective. Remission is maintained in $80-90 \%$ of patients. However, in order to prevent relapse, it is also possible to switch to AZA after induction therapy with Cyp after 3-6 months, at an initial AZA dose of $2 \mathrm{mg} / \mathrm{kg} / \mathrm{die}$. This can be reduced to $1.5 \mathrm{mg} / \mathrm{kg} /$ die after a year of starting treatment. ${ }^{32} \mathrm{AZA}$ has been widely used as maintenance therapy in Europe and is metabolized into thiopurine nucleotides by the liver. This interferes with the enzymes necessary for purine synthesis leading to reduced cell proliferation. Prolonged use of AZA can result in an increased risk of squamous cell skin carcinoma.

In one open, multi-center prospective study, Pagnoux et al. reported that patients with WG or MPA remission were assigned to receive AZA ( $2 \mathrm{mg} / \mathrm{kg} / \mathrm{die})$ or MTZ $(0.3 \mathrm{mg} / \mathrm{kg} /$ week gradually increasing to 25 $\mathrm{mg} /$ week) for 12 months. Of the 159 patients selected to take part in the study, $126(79 \%)$ were in remission. Patients were randomly allocated to two groups of 63 patients each. Each group was assigned to receive either AZA or MTX and followed up for an average 29 months. Adverse events were experienced by 29 patients treated with AZA and in 35 patients treated with
MTX ( $\mathrm{P}=0.29)$. Grade 3 or 4 adverse events were observed in 5 patients in the AZA group and in 11 in the MTX group $(\mathrm{P}=0.11)$. Twenty-three patients treated with AZA and 21 treated with MTX relapsed $(\mathrm{P}=0.71)$. In $73 \%$ of cases, patients relapsed after interruption of treatment during the study phase. The two drugs seemed to be equivalent for maintenance therapy in patients with WG or MPA.

\section{Leflunomide}

Leflunomide is an oral alternative to AZA or MTX for maintenance in disease relapse. Leflunomide offers a new therapeutic approach and an alternative oral treatment to AZA or to MTX that is used in the maintenance of disease relapse. Its mechanism of action is not fully understood but may be related to its ability to inhibit de novo pyrimidine biosynthesis through the inhibition of the dihydroorotate dehydrogenase enzyme. ${ }^{33}$ It has been well tollerated even though rather high doses are required $(30 \mathrm{mg} / \mathrm{die})$ compared with the dosage used in the treatment of AR. During 2-year follow up, $45 \%$ of patients relapsed while the most frequent side effects were respiratory infections and onset of arterial hypertension. ${ }^{34}$

Leflunomide has been shown to be more effective in maintaining remission than MTX but with excessive side effects. Lower dosing regimens have yet to be evaluated.

Besides immunosuppressors, also trimethoprim/ sulfamethoxazole can be used in WG to prevent relapse. The rationale for its use is based on some positive results on a limited number of cases, and on the increased frequency of relapse in those WG patients who present chronic nasal infection.

\section{Cyclosporine}

Cyclosporine has been used less as maintenance therapy. It is mainly used as second choice treatment in cases of Cyp intollerance or in those patients undergoing kidney transplant, both on its own or in combined therapy.

\section{Endovenous immunoglobulin 5}

Some small studies have attempted to evaluate the immunosuppressor potential of high doses of endovenous Ig on patients with WG. However, for the moment, few data are available. In general, treatment is continued for 1-2 years after stable remission is achieved.

\section{Treatment of relapse}

In patients with WG treated with Cyp and PD, relapse occurs in $20-45 \%$. Risk factors for relapse include the habit of using corticosteroids on their own, a reduc- 
tion in therapies that are too rapid, additional infections and an increase in ANCA. Relapse usually occurs within the first year after suspension of the immunosuppressive therapy. Disease that does not respond to induction treatment with Cyp and steroids or that does not respond to treatment or standard maintenance is considered refractory. While true refractory disease is unusual, up to $50 \%$ of patients with AVV will relapse over a 5-year period. ${ }^{5}$ Relapse rates are higher with patients with WG and in those with PR3-ANCA positivity.

A 4-fold rise in ANCA titer has been found to be predictive of relapse but with a variable time interval (mean 5-8 months, range 2-12 months). In the same study, 6 of 48 patients experienced relapse while ANCA titers were negative or falling. There have been attempts to treat rising ANCA titers preemptively with increased immunosuppression to avoid relapse. However, in practice, this could lead to overtreatment and thus our approach is close observation of rising ANCA titers and not to start immunosuppression. In those patients undergoing immunosuppressor therapy, an increase in dosage of the immunosuppressors and the corticosteroids can first be attempted while in cases in which therapy has been suspended, or in those cases with serious relapse, the initial induction therapy needs to be restarted. However, consideration should be given to the increased exposure to cytotoxic drugs since treatment after relapse must be continued for at least two years. Furthermore, if the patient relapses during maintenance therapy other different drugs should be used.

\section{New therapeutic approaches}

Mycophenolate mofetil (MMF) (2 g/die) inhibits inosine monophosphate, an enzyme necessary for purine synthesis, which results in the inhibition of Tand B-lymphocyte activity. A small study in China ${ }^{34}$ reported more favorable results in achieving remission rates and maintaining normal renal function at six months in patients treated with MMF (44\%) than in those treated with Cyp. ${ }^{35}$ According to the EUVAS, MMF should be more widely used in maintenance treatment. MMF is now routinely used in long-term treatment to prevent allograft rejection in renal transplant patients because it has been proven to be superior to AZA. ${ }^{36}$ Consequently, there has been considerable interest in the use of MMF as a maintenance agent in place of AZA in vasculitis.

\section{Calcineurin inhibitors}

Cyclosporin and tacrolimus are both calcineurin inhibitors, which inhibit T-lymphocyte signal transduction and IL-2 transcription. Cyclosporin was historically the mainstay of immunosuppression for renal transplantation. Recently, tacrolimus has been shown to be efficient in preventing rejection in transplant patients, being widely superior to cyclosporine.

\section{Treatment of relapsing or refractory disease}

Despite their extensive use in transplantation, and in primary glomerulonephritis, the role of these drugs in systemic autoimmune diseases has not been systematically validated and there are no randomized trials using calcineurin inhibitors in AAV. Their potential usefulness may be inferred from the low relapse rates in transplantation, and successful use has been reported in case reports and small cohort studies in AAV. Cyclosporin has been used as second-line maintenance therapy in systemic lupus erythematosus (SLE) in uncontrolled trials in small numbers of patients with some effect and tacrolimus has been used in induction of remission in renal SLE. However, recent collaborative trials in $\mathrm{AAV}$ have focused on evaluating the role of other agents such as MMF and RTX.

Perhaps surprisingly, MMF was inferior to AZA in the maintenance of remission in AAV with time to relapse as the primary end point. Therefore, it seems unlikely that MMF will be used as a standard alternative to AZA in maintenance, although it could be used as a second-line agent in relapsing disease or in those intolerant to AZA.

\section{Biological therapies: anti TNF- $\alpha$}

New prospectives regard the use of anti TNF- $\alpha$ in WG, at least in those refractory forms that do not respond to standardized therapy. It is known that TNF$\alpha$ intervenes in activating different cell populations involved in the immuno-inflammatory processes, and, in particular, plays an important role in the formation of granulomatose lesions and in inducing vasculitis.

Infliximab is a chimeric monoclonal antibody that binds soluble and transmembrane forms of TNF$\alpha$. It has demonstrated efficacy in induction and in maintenance therapies in patients in remission if used together with conventional therapy. It reduces the need for steroids but increased the risk of serious infection.

Etanercept is a fusion protein consisting of two extracellular p75 TNF- $\alpha$-receptor domains linked to the Fc portion of human IgG1. A randomized controlled trial of etanercept in 180 patients with WG, mainly without severe renal disease, showed no benefit in the induction or maintenance of remission (although prednisolone was withdrawn at six months) and demonstrated a high rate of treatment-associated complications and excessive solid organ tumors. It is not clear why these two mechanisms of TNF- $\alpha$ blockade gave rise to such different outcomes, and this may relate to their different biological effects. No efficacy in maintaining remission has been demonstrated. ${ }^{37}$ Data 
available involve small numbers of patients treated with standard induction therapy (oral $\mathrm{Cy}+\mathrm{PD}$ ) or with other immunosuppressors (AZA, MTX) with the addition of infliximab (6 infusions of $5 \mathrm{mg} / \mathrm{kg}$ at Weeks $0,2,6,12$, $18,24)$. In the majority of cases complete or partial remission was achieved but various adverse events were observed: frequent and severe respiratory infections and marked lymphocytopenia. Given this, future treatment strategies should consider a reduction in dosage of monoclonal antibody $(3 \mathrm{mg} / \mathrm{kg})$, bolus administration of Cyp, phrophylaxis with trimethoprim/sulfamethoxazole. A possible role for etanercept in the treatment of ANCA-associated vasculitis should also be evaluated.

\section{B-cell depletion}

Successful results have been reported in B-cell depletion with the anti-CD20 chimeric monoclonal antibody rituximab (RTX) in refractory disease. It has been used as an induction agent in cases of remission. Comparative studies have been carried out with Cyp and have shown similar remission rates. A longer evaluation should be carried out to examine relapse rates after Bcell reconstitution. ${ }^{38,39}$

\section{Other therapies for refractory disease}

Other monoclonal antibodies have been used in recent studies of small patient numbers and these should be confirmed in the future: i) alemtuzamab is a humanized monoclonal antibody that specifically binds to $\mathrm{CD}$ 52 on the surface of lymphocytes and monocytes ${ }^{40}$ in patients with WG; ii) omalizumab in CSS; iii) antithimocyte globulin (ATG) is made up of polyclonal antibodies directed against antigens of the surface of activated $\mathrm{T}$ cells. It is used in B-cell lymphoma, allogeneic stem cell transplantation, and renal transplantation; iv) anti-IL-5 antibodies; v) endovenous immunoglobulin has been used to induce remission with significant success ${ }^{41}$ either as sole therapy or for its treatment-sparing effect. Patients with chronic relapse require the addition of other off-label therapies such as infliximab or RTX. Greater caution should, therefore, be exercised with these treatments, and clinical experience and authorized administration approval are needed.

\section{Monitoring for complications}

Careful monitoring is mandatory to minimize morbidity and mortality, and to effectively manage therapy. Infection, drug toxicity, or a new disease can all cause a worsening of the clinical condition. Infection is the main cause of morbidity and mortality $(10 \%$ serious infections). Drug toxicity is a relatively frequent problem and is of clinical importance. Relapse is frequent and occurs in $50 \%$ of cases in WG, sometimes with different signs and symptoms, and more severe symptoms are also observed with involvement of new organ systems. The success of RTX has led to the development of further agents for B-cell depletion.

\section{Conclusions}

Relapse has also been associated with nasal carriage of Staphylococcus aureus in WG and consequently our practice is to maintain most patients with WG on long-term prophylactic cotrimoxazole, ${ }^{29}$ although care needs to be taken with concomitant use of MTX.

\section{References}

1. Leavitt RY, Fauci AS, Reinhold-Keller E, et al. Problematic granulomatous lung. Am Rev Respir Dis 1986;134:149-66.

2. Cees GM, Kallemberg CG. Antineutrophil cytoplasmic autoantibody. Curr Opin Rheumatol 2007;19:17-24.

3. Jennette JC, Falk RJ, Andrassy K, et al. Nomenclature of systemic vasculitides. Proposal of an international consensus conference. Arthritis Rheum 1994;37:187-92.

4. Stegeman CA, Branten JW, Reichert LJ, et al. Pathogenesis and treatment of ANCA-associated vasculitides: Are MPO-ANCA and PR3-ANCA pathogenic? Ann Intern Med 1994;335:16-20.

5. Kallemberg CG. Churg-Strauss syndrome with poorprognosis factors: a prospective multicenter. Curr Opin Rheumatol 2007;19:17-24.

6. Collins CE, Quismorio FP Jr. Pulmonary involvement in microscopic polyangiitis. Curr Opin Pulm Med 2005; 11:447-51.

7. Booth AD, Almond MK, Burns A, et al. Outcome of ANCA-associated renal vasculitis: A 5-year. Plaisance Retrospective Study. Am J Kidney Dis 2003;41:776-84.

8. Walton EW. Giant-cell granuloma of the respiratory tract (Wegener's granulomatosis). BMJ 1958;2:265-70.

9. Falk RJ, Gross WL, Guillevin L, et al. An alternative name for Wegener's granulomatosis. Ann Rheum Dis 2011;70:704.

10. Matteson EL, Gold KN, Bloch DA, et al. Long-term survival of patients with Wegener's granulomatosis from the American College of Rheumatology Wegener's Granulomatosis Classification Criteria Cohort. Am J Med 1996;101:129-34.

11. Kallenberg CG, Heeringa P, Stegeman CA. Mechanisms of Disease: pathogenesis and treatment of ANCA-associated vasculitides. Nat Clin Pract Rheumatol 2006;2: 661-70.

12. Frankel SK, Reinhold-Keller E, Herlyn K, et al. The diagnosis and treatment of pulmonary vasculitis. Chest 2006; 129:452-65.

13. Thickett DR, Richter AG, Nathani N, et al. Pulmonary manifestations of antineutrophil cytoplasmic. Rheumatology 2006;45:261-8.

14. Mansi IA, Opran A, Rosner F. ANCA-associated smallvessel vasculitis. Am Fam Physician 2002;65:1615-20.

15. Brown K. Pulmonary vasculitis. Proc Am Thorac Soc 2006;3:48-57.

16. Westman KW, Bygren PG, Olsson H, et al. Relapse rate, 
renal survival, and cancer morbidity in patients with Wegener's granulomatosis or microscopic polyangiitis with renal involvement. J Am Soc Nephrol 1998;9:842-52.

17. Frankel SK, Reinhold-Keller E, Herlyn K, et al. Update in the diagnosis and management of pulmonary vasculitis. Chest 2006;129:452-65.

18. Alberts WM. Pulmonary manifestations of the ChurgSrauss. Curr Opin Pulm Med 2007;13;445-50.

19. Bosch X, Guilabert A, Espinosa G, Mirapeix E. Treatment of antineutrophil cytoplasmic antibody associated vasculitis: a systematic review. JAMA 2007;298:655-69.

20. Occhipinti G, Bartolomei C. Classificazione ed Epidemiologia delle Vasculiti. XIII Congresso Nazionale FADOI, Firenze, Italy, 4-7 May, 2008.

21. Corrao S, Pistone G. Manuale pratico per la diagnosi in reumatologia. Roma: Pensiero Scientifico Editore; 2009. pp 93-99.

22. Choi YH, Im JG, Han BK, et al. Thoracic manifestations of Churg-Strauss syndrome. Chest 2000;117,24-6.

23. Rutgers AP, Heeringa JW, Tervaert C. The role of myeloperoxidase in the pathogenesis of systemic vasculitis. Clin Exp Rheumatol 2003;21 Suppl 32:S55-63.

24. Pagnoux C, Loic G, Guilpain P. Churg-Strauss syndrome. Curr Opin Rheumatology 2007;19:25-32.

25. Exley AR, Bacon PA, Luqmani RA, et al. Development and initial validation of the Vasculitis Damage Index for the standardized clinical assessment of damage in the systemic vasculitides. Arthritis Rheum 1997; 40:371-80.

26. Falk RJ, Hoffman GS. Controversies in small vessel vasculitis. Curr Opin Rheumatol 2007;19:1-9.

27. Thickett DR, Richter AG, Nathani N, et al. Pulmonary ANCA-associated vasculitis have suggested that pulmonary involvement is associated. Rheumatology 2006;45:261-8.

28. Little MA, Pusey CD. Rapidly progressive glomerulonephritis: current and evolving treatment strategies. J Nephrol 2004; 17:10-9.

29. Hamour S, Salama AD, Pusey CD. Management of ANCA-associated vasculitis: current trends and future prospects. Ther Clin Risk Manag 2010;6:253-64.

30. Riccieri V, Valesini G. Terapia della granulomatosi di Wegener. Reumatismo 2004:56:69-76.

31. Jayne D, Rasmussen N, Andrassy K, et al. A randomized trial of maintenance therapy for vasculitis associated with antineutrophil cytoplasmic autoantibodies. N Engl J Med 2003;349:36-40.

32. Hogan SL, Falk RJ, Chin H, et al. Predictors of relapse and treatment resistance in antineutrophil cytoplasmic antibody-associated small-vessel vasculitis. Ann Intern Med 2005;143:621-31.

33. Pagnoux C, Mahr A, Hamidou MA, et al. Value of antiinfective chemoprophylaxis in primary systemic vasculitis: what is the evidence? $\mathrm{N}$ Engl $\mathrm{J}$ Med 2008;359:2790-803.

34. Metzler C, Miehle N, Manger K, et al. Elevated relapse rate under oral methotrexate versus leflunomide for maintenance of remission in Wegener's granulomatosis. Rheumatology (Oxford) 2007;46:1087-91.

35. Hu W, Liu C, Xie H, et al. Mycophenolate mofetil versus cyclophosphamide for inducing Remission. Nephrol Dial Transplant 2008;23:1307-12.

36. Hiemstra T, Walsh M, Mahr A, et al. Mycophenolate mofetil vs associated vasculitis: a randomized controlled trial. JAMA 2010;304:2381-8.

37. Wegener's Granulomatosis Etanercept Trial Research Group. Etanercept plus standard therapy for Wegener's granulomatosis. N Engl J Med 2005;352:351-61.

38. Jones R, Tervaert JW, Hauser T, et al. Randomized trial of rituximab vs cyclophosphamide for ANCA-associated renal vasculitis: RITUXVAS. Proceedings of the 14th International Vasculitis and ANCA workshop, 6-9 Jun, 2009. Lund Sweden. APMIS 2009;117 Suppl 127:A22.

39. Lockwood CM, Thiru S, Stewart S, et al. Treatment of refractory Wegener's granulomatosis with humanized monoclonal antibodies. QJM 1996;89:903-12.

40. Walsh M, Chaudhry A, Jayne D. Long-term follow-up of relapsing/refractory anti-neutrophil cytoplasm antibody associated vasculitis treated with the lymphocyte depleting antibody alemtuzumab (CAMPATH-1H). Ann Rheum Dis 2008;67:1322-7.

41. Martinez V, Cohen P, Pagnoux C, et al. Intravenous immunoglobulins for relapses of systemic vasculitides associated with antineutrophil cytoplasmic autoantibodies: Results of a multicenter, prospective, open-label study of twenty-two patients. Arthritis Rheum 2008;58:308-17. 